\title{
Untersuchungen über die Physiologie der Lymphbewegung.
}

\author{
Von \\ Seigo Funaoka. \\ Aus dem Anatomischen Institut der Kaiserlichen Universität zu Kyoto.
}

\section{Wie viel Lymphe ist zur Anfüllung einer Lymphdrïse nötig?}

Von

\section{Yoshiteru Takabatake.}

Mit 3 Textfiguren.

Dảe Passe der Lymphflüssigkeit in den Lymphknoten wurde von Frey ${ }^{1)}$ und $\mathrm{His}^{2)}$ schon vor langem klargelegt. Die, durch die Vasa afferentia zugeleitete Lymphflüssigkeit strömt unter nur schwachem Drucke in den Lymphsinus ein und bewegt sich in diesem langsam. von der Peripherie her gegen den Hilus hin. Der Druck, unter dem die Flüssigkeit gewöhnlich in den Sinus eintritt, genügt nicht zum Weitertreiben von hier aus in die Drüsensubstanz. Im Gegenteil geht ein Flüssigkeitsstrom aus der von Gefässen reichlich durchzogenen Drüsensubstanz nach aussen, d. h. nach dem Sinus hin, um sich von da aus in die Vasa efferentia zu ergiessen.

Die Kapazität des ganzen Sinus eines Lymphknotens hängt von der

1) Frey, H.: Zeit. f. wiss. Zool. Bd. 13, S. 28, 1863.

2) His: Zeit. f. wiss. Zool. Bd. 11, S. 65, 1861. 
Spannung seiner Kapsel ab. In gewöhnlichem Zustande des Lymphknotens muss aber diese Spannung unbedeutend sein, da der periphere

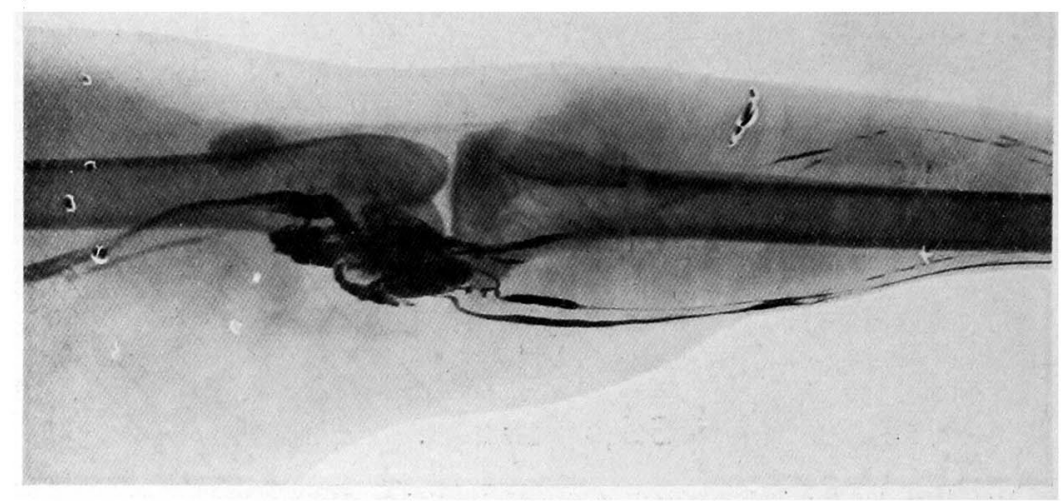

Fig. 1 .

Lymphdruck, wie Funaoka ${ }^{1)}$ mit Tachikawa zusammen an Kaninchen mass, nur sehr gering ist, nämlich unter $15 \mathrm{~mm} \mathrm{Hg}$ beträgt. Die einem Lymphknoten zugeleitete Flüssigkeit geht also langsam durch die Sinusräume hindurch, ohne Anschwellung des Knotens zu verursachen, wemn die Passage in den ableitenden Gefässen nicht gestört ist.

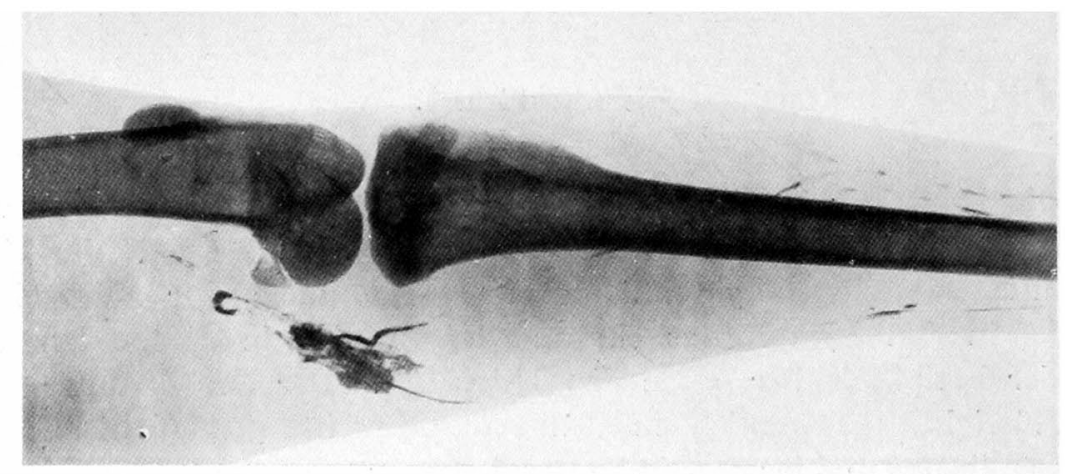

Fig. 2.

Die Kapazität eines Knotens, die ungefähr dem Gesarntlumen aller Sinusräume entspricht, kann dúrch die Menge der in ihm strömenden Flüssigkeit gemessen werden. Wir injizierten daher Kaninchen in die

1) Funaoka u. Tachikawa: Arbeiten aus der III. Abt: des anatomischen Inst. Kaiserl. Universität. Serie D. Heft 1, 1930. 
Fusssohle eine kleine Menge von Di-n-propylquecksilber ${ }^{1)}$, röntgenographierten den Knieteil und konstatierten nach sanfter Massage der Fusssohle das. Durchgehen der Kontrastmasse durch die Lymphogl. poplitea in die Vasa femoralia. Wir nahmen nach diesem Röntgenbefund an, dass die Sinusräume mindestens zum grössten Teile mit dem Quecksilberpräparat ausgefüllt waren. Wir schnitten darauf den Lymphknoten aus und stellten mittels chemischer Analyse die Quecksilbermenge in demselben fest.

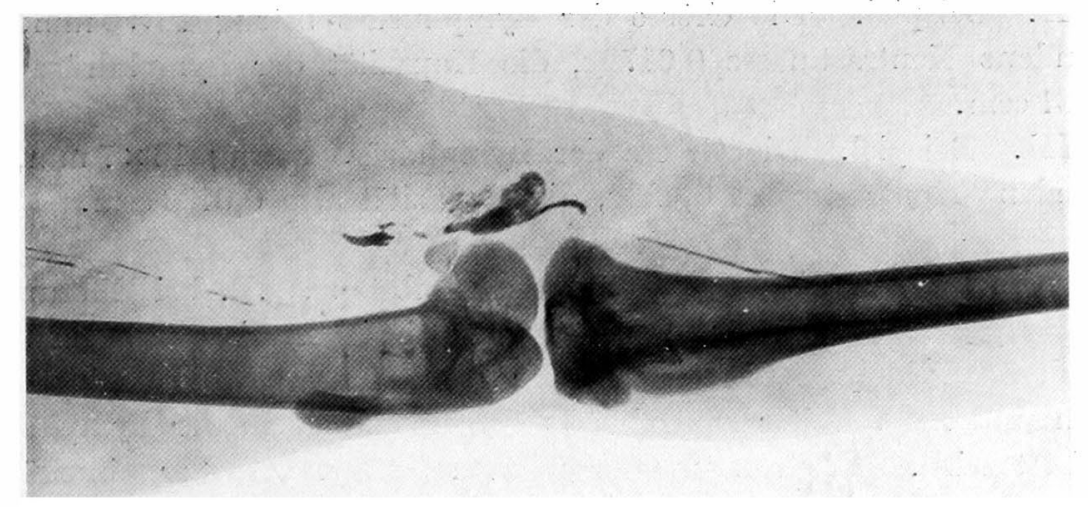

Fig. 3.

Um die Methode zu veranschaulichen mag ein Beispiel folgen.

I. Beispiel.

1) In die Fusssohle eines mittelgrossen Kaninchens wurde etwa $1 \mathrm{ccm}$ Di-n-propylquecksilber injiziert.

2) Nach einigen Minuten wurde mittels Röntgenstrahlen das Durchgeherr der Kontrastmasse durch die Kniedrüse festgestellt.

3) Die Lymphdrüse wurde ausgeschnitten. Ihre Grösse betrug $22 \times 11 \mathrm{~mm}$.

4) Die ganze Lymphdrüse wurde mit etwa $20 \mathrm{ccm}$ Säuregemisch unter Erwärmen verflüssigt und diese Flüssigkeit mit Ammoniak neutralisiert.

5) Nach dem Ansäuern der filtrierten Flüssigkeit mit $\mathrm{ClH}$ wurde $\mathrm{SH}_{2}$ eingeleitet, um $\mathrm{SHg}$ sich absetzen zu lassen.

6) Das gesammelte $\mathrm{SHg}$ wurde in einigen ccm Königwasser aufgelöst, mit $\mathrm{NH}_{4} \mathrm{OH}$ neutralisiert, angesäuert und das $\mathrm{SHg}$ sich wieder absetzen gelassen.

1) Fujita: Arbeiten aus der III. Abteilung des anatomischen Instituts der Kaiserl. Unitersität Kyoto. Serie D. Heft. 1, 1930. 
7) Darauf wurde mit dem Goochschen Tiegel filtriert, getrocknet und gewogen.

Auf diese Weise erhielten wir aus der Lymphdrüse 0,0772g $\mathrm{SHg}$. Diese Menge SHg entspricht 0,0952g Di-n-propylquecksilber. Da das spezifische Gewicht der Kontrastmasse, 2,124 beträgt, so ist das Volumen der gefundenen Kontrastmasse :

$$
0,0952 \div 2,124=0,0448 \mathrm{ccm}
$$

II. Beispiel. Die Grösse der Lymphdrüse betrug $16 \times 6 \mathrm{~mm}$, die gefundene Kontrastmasse $0,0172 \mathrm{~g}$, die Kapazität der Lymphdrüse also $0,0081 \mathrm{ccm}$.

III. Beispiel. Die Grösse der Lymphdrüse betrug $16 \times 7 \mathrm{~mm}$, die gefundene Kontrastmasse $0,022 \mathrm{~g}$, die Kapazität also $0,0103 \mathrm{ccm}$.

Beim raschen Eintreten von giftiger Substanz, z.B. bei Eiterung in der Peripherie kamn nach den obigen Beispielen diese Substanz die unbedeutend kleinen Sinusräume der lokalen Lymphdrüse leicht ausfüllen und noch weiter zentralwärts gehen, ehe sie in der Lymphdrüse entgiftet wird. Die von uns gefundenen Lumenwerte der Sinusräume stellen sicherlich Minima dar, da die Kontrastmasse unterwegs bis zur Drüse durch die Lymphflüssigkeit verdünnt und ihr wahrscheinlich in der Lymphdrüse selbst aus dem Blut ausgeschwitzte Flüssigkeit beigemischt wird. 